\title{
Mu to electron conversion with the COMET experiment
}

\author{
Peter Dornan \\ Imperial College London, United Kingdom
}

\begin{abstract}
The COMET experiment at J-PARC is under construction and will search for mu to electron conversion in aluminium at the $3.10^{-17}$ level, four orders of magnitude better than current limits. The experiment will take place in two phases with a first phase aiming at a two orders of magnitude improvement.
\end{abstract}

\section{Charged lepton flavour violation with COMET}

The COMET experiment is currently in preparation at $\mathrm{J}$ PARC. The aim is to investigate charged lepton violation by searching for $\mathrm{mu}$ to e transitions from muons stopped in aluminium. The collaboration currently has 177 participants from 33 institutes from Belarus, Canada, China, Czech Republic, France, Georgia, Germany, India, Japan, Malaysia, Russia, Saudi Arabia, South Korea, United Kingdom and Vietnam. First data taking is expected in 2018.

Lepton flavour violation does not feature in the standard model which only describes flavour violations in the charged current interactions of quarks. However neutrino oscillation shows that neutral lepton flavour violation does occur in nature and this, so far, is the one unambiguous observation in particle physics of an interaction beyond the basic standard model. The actual mechanism of lepton flavour violation in the neutrino sector remains to be discovered and so establishing if there is measurable lepton flavour violation in the charged sector is crucial in the search for a wider theory or at least the necessary extension of the standard model to encompass neutrino oscillation.

Flavour is a crucial part of particle physics and whilst it is becoming increasingly well parameterised there is little real appreciation of its role. Consequently it is necessary to acquire as much information as possible if a valid theory is to emerge. An important consequence of flavour changing processes with three families is the mixing matrix with the crucial phase which can yield CP violating processes. Observation of charged lepton flavour processes will almost certainly lead to additional sources of CP violation which could contribute to the unexplained baryon - antibaryon asymmetry.

\subsection{Mu to e transitions}

A search for a muon changing to an electron is the transition most accessible for experimental investigation of charged lepton violation. Of course it could be that the main transition is from the tau but the lower production rate and the very short lifetime make it much harder to achieve very low rates. Three processes for $\mu$ to e transitions are currently being investigated

$$
\begin{gathered}
\mu \rightarrow e \gamma \\
\mu \rightarrow e e e \\
\mu+N \rightarrow e+N
\end{gathered}
$$

Present limits are currently: $\operatorname{Br}(\mu \rightarrow$ e $\gamma)<5.7 .10^{-13}$, $\operatorname{Br}(\mu \rightarrow$ eee $)<1.10^{-12}$ and $\mu$ to e conversion on gold lower than $6.10^{-13}$. Currently $\mu \rightarrow$ e $\gamma$ and $\mu \rightarrow$ eee are being investigated at PSI. Two experiments are in construction to investigate $\mu$ to e conversion, COMET at J-PARC and Mu2e at FNAL.

As a result of neutrino oscillation charged lepton violation must take place at some level This is shown in Figure 1, however the rate from this process is very small, $\sim\left(\mathrm{m}_{\mathrm{v}} / \mathrm{m}_{\mathrm{W}}\right)^{4}$ and so effectively unobservable.

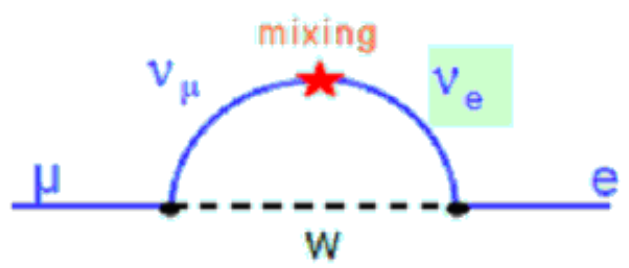

Figure 1. Mu to e transition as a result of neutrino oscillation.

Possible processes for charged lepton violation can be classified as 'Photonic', Figure 2, and Non-Photonic, Figure 3.
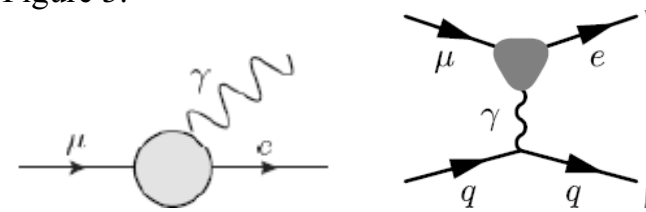

Figure 2. Photonic processes for $\mu$ to e Transitions
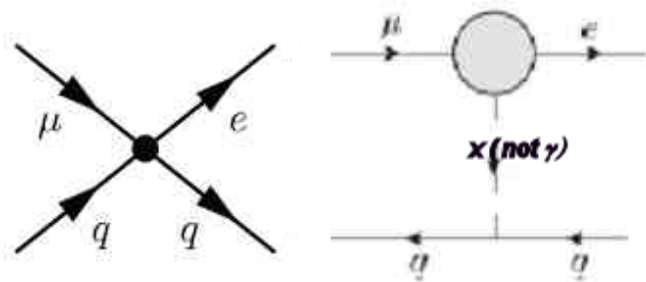

Figure 3. Non-photonic processes for $\mu$ to e Transitions

These can be summarised by a Lagrangian[1] 


$$
\begin{array}{r}
\mathcal{L}_{\mathrm{CLFV}}=\frac{m_{\mu}}{(\kappa+1) \Lambda^{2}} \bar{\mu}_{R} \sigma_{\mu \nu} e_{L} F^{\mu \nu}+\text { h.c. } \\
\frac{\kappa}{(1+\kappa) \Lambda^{2}} \bar{\mu}_{L} \gamma_{\mu} e_{L}\left(\bar{u}_{L} \gamma^{\mu} u_{L}+\bar{d}_{L} \gamma^{\mu} d_{L}\right)+\text { h.c. }
\end{array}
$$

where $\kappa$ gives the relative strength of photonic and non-photonic processes and $\Lambda$ is the scale of new physics. For only photonic processes $\kappa=0$ and the $\mu$-e conversion rate is $\sim 1 / 100$ of $\mu \rightarrow \mathrm{e} \gamma$, however if there are only non-photonic processes, $\kappa \rightarrow \infty$ and then only $\mu$-e conversion and $\mu \rightarrow$ eee will occur.

\section{Experimental Aspects of $\mu$ to $\mathrm{e}$ Conversion}

The major problem for experiments seeking to observe and measure very rare events is the elimination of backgrounds. Two aspects of coherent $\mu$ to e conversion off a nucleus produce a very characteristic signal which enables high background suppression. These are the energy of the emitted electron and the time following the production of the muon to the electron appearance.

In $\mu$ to $\mathrm{e}$ conversion the emitted electron is monoenergetic and equal to the muon mass minus the binding energy and nuclear recoil. This comes to 105 $\mathrm{MeV}$ for conversion in aluminium. The electron is released after the muon is stopped in the aluminium target and so there is a time delay following the muon production given by the lifetime of a captured muon in aluminium, which is $864 \mathrm{nsec}$. The signal is therefore an identified electron with $105 \mathrm{MeV}$ energy in a specific time interval following muon production. A pulsed proton beam is used to generate the muons, therefore a sufficient gap between pulses and very high extinction between pulses are essential. The beam timing setup for COMET is shown in Figure 4.

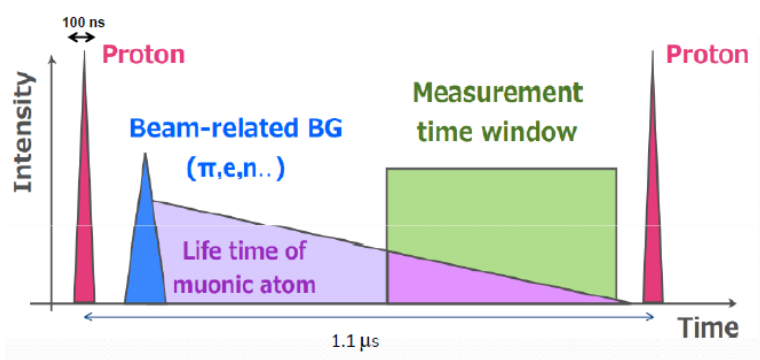

Figure 4. Proton pulses from J-PARC for COMET are separated by $1.1 \mu \mathrm{sec}$ and the time window for measurement is typically from 0.6 to $1 \mu \mathrm{sec}$.

\section{The COMET Experiment}

The essential features of COMET are shown diagrammatically in Figure 5. An intense $56 \mathrm{~kW} 8 \mathrm{GeV}$ proton beam is incident on a target in a $5 \mathrm{~T}$ magnetic field. This forms the pion capture section where soft backward going pions are trapped and directed to the decay and muon transport curved solenoids. The pions quickly decay to muons which are transported and momentum selected by curved solenoids and collimators to be captured in thin aluminium disks. These form the muon stopping target where the mu to electron conversion will take place. Electrons emitted from the conversion process are then transported by further momentum selecting curved solenoids to the electron detector, StrECal. The goal is a single event sensitivity of $3.10^{-17}$ for $\mu$ to e conversion.

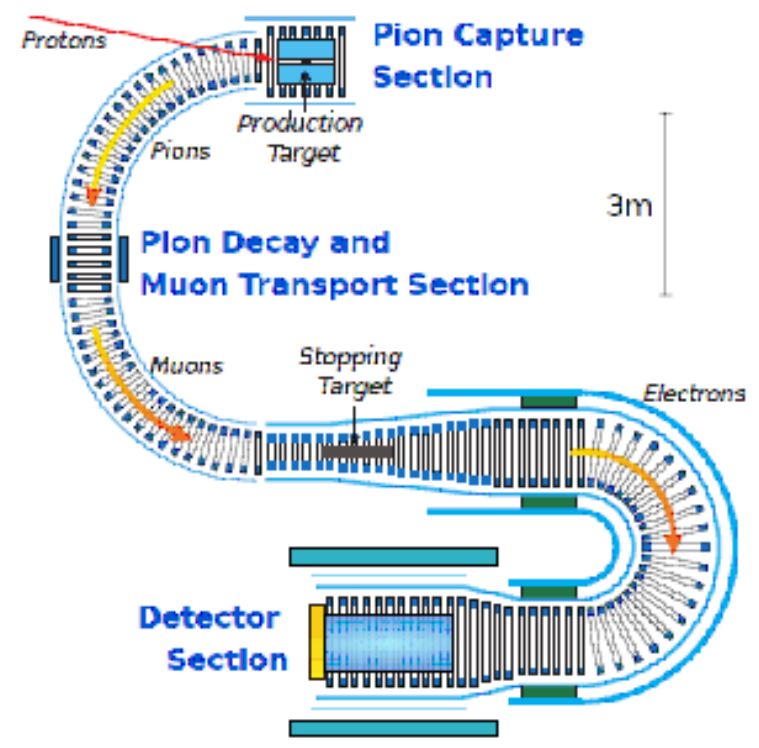

Figure 5 Outline of the COMET Experiment at J-PARC.

\subsection{The Curved Solenoids}

A critical feature of the experiment is the use of curved solenoids for momentum selection and background minimisation. However in a curved solenoid there is drift in the beam position from the solenoid centre for the nominal momentum as shown in Figure 6 and this requires correction by means of an additional vertical dipole field.

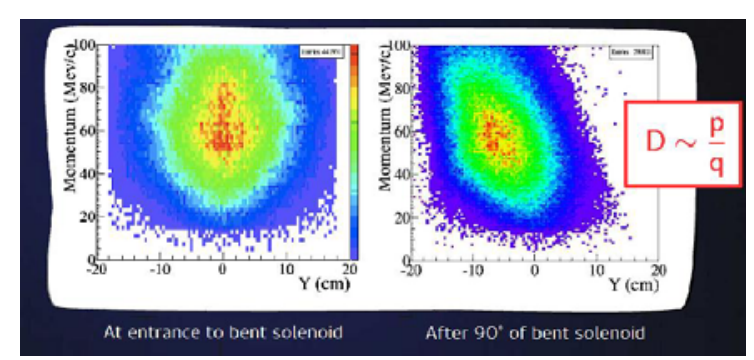

Figure 6. Simulation of the drift of the beam after a $90^{\circ}$ transport in the bent solenoid dependent on the momentum, $p$, and the charge, $q$.

The first curved solenoid was delivered to J-PARC from Toshiba in March 2015 as shown in Fig. 7 


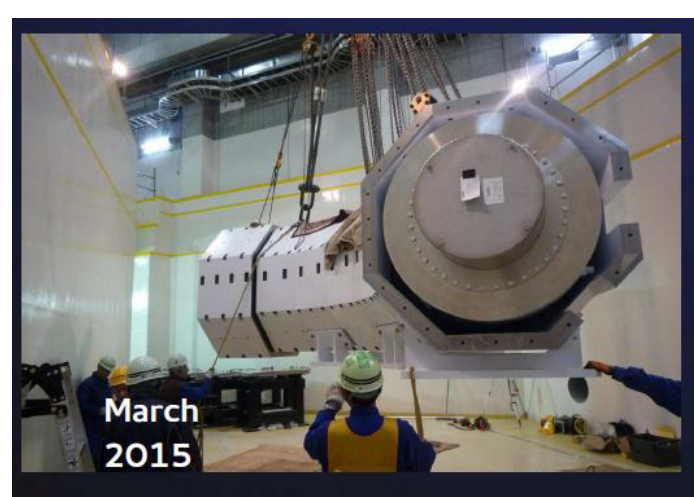

Figure 7. Delivery of the first curved solenoid to J-PARC for the COMET experiment.

\subsection{The Electron Detector, StrECal}

The electron transport and StrECal detector are shown diagrammatically in Figure 8.

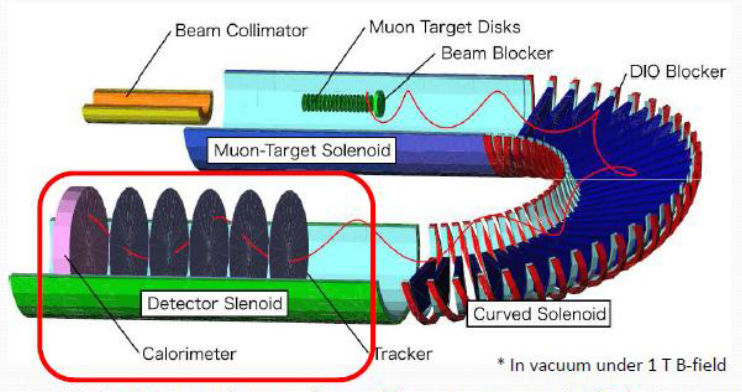

StrECal= Straw tube tracker + Electro-magnetic Calorimeter

Figure 8. Layout of the electron transport and the elctron detector, StrECal.

The StrECal consists of a straw tube tracker in front of an electromagnetic crystal calorimeter in a $1 \mathrm{~T}$ solenoid. The straw tracker will have a minimum of 5 modules each with 4 planes, 2 in $\mathrm{x}$ and 2 in $\mathrm{y}$. The exact number is still being optimised. These planes will be installed in vacuum to minimise multiple scattering and provide a spatial resolution $<200 \mu$ to give a momentum resolution $<200 \mathrm{keV} / \mathrm{c}$ for a $105 \mathrm{MeV}$ track. For the ECAL the requirements are an energy resolution better than $5 \%$ at $100 \mathrm{MeV}$ and a spatial resolution better than $1 \mathrm{~cm}$. This requires a crystal calorimeter and two crystals GSO and LYSO have been examined in prototypes. The results are shown in Fig 9 and as a result the LYSO crystals have been chosen.

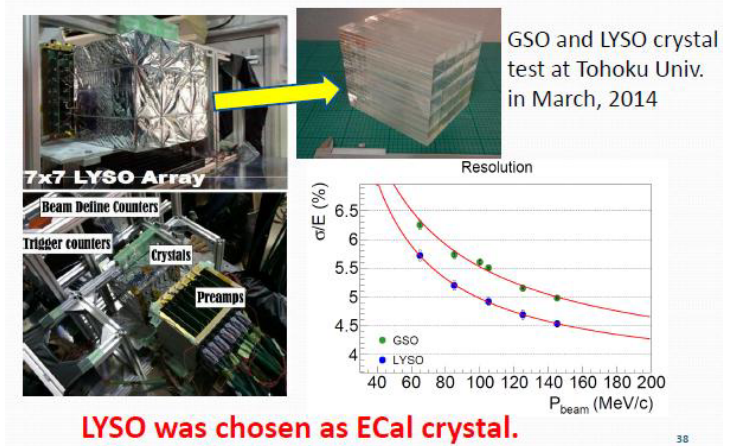

Figure 9. Calorimeter prototyping leading to the choice of LYSO for the COMET ECAL.

\section{Backgrounds}

Understanding and minimising backgrounds are critical for a very low rate measurement. Some of the most important for COMET are described.

\subsection{Muon Decays in Orbit (DIO)}

Standard muon decays to evv occur whilst the muon orbits the nucleus. These produce electrons with the same time structure as the signal although with a softer momentum spectrum, emphasising the importance of the momentum-energy resolution of the detector. Figure 10 shows the upper part of the expected DIO spectrum together with a signal corresponding to a $\mu$ to $\mathrm{e}$ conversion branching ratio of $3.10^{-15}$.

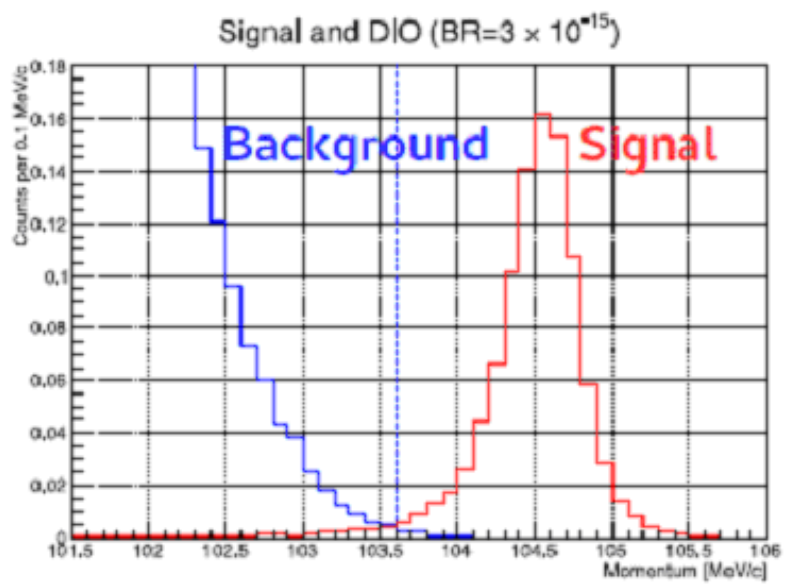

Figure 10. Momentum spectrum expected for the DIO background and a signal of $3 \cdot 10^{-15}$.

\subsection{Out of Time Protons on the Target}

After the protons hit the target there is a prompt burst of particles which would swamp any signal and this is eliminated with the use of the time window as shown in Figure 4. However should there be additional protons between the bunches these can cause serious backgrounds within the signal time window and so it is crucial that the accelerator has a high extinction level requiring an extinction factor

Extinction Factor $=\frac{\text { No. of protons between bunches }}{\text { No of protons in the bunch }}<10^{-10}$.

This has now been achieved at J-PARC as shown in Figure 11.

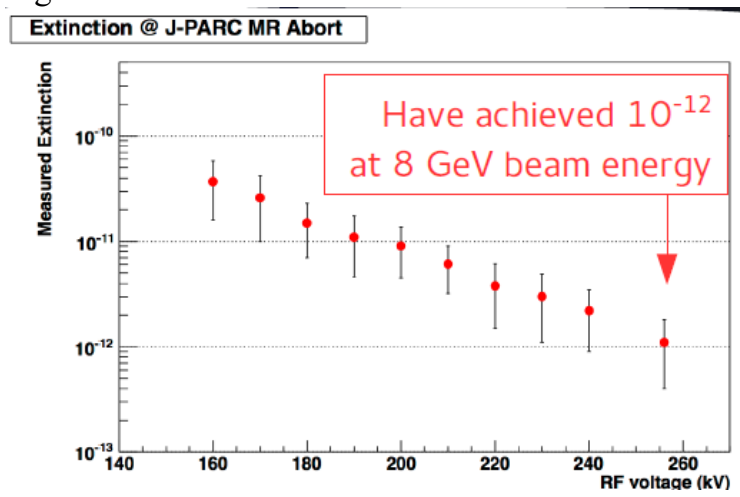

Figure 11. Extinction levels reached in the $8 \mathrm{GeV} \mathrm{J-PARC}$ beam 


\subsection{Other Particles Emitted when a Muon is captured by Aluminium}

At the level of accuracy required, predictions are unreliable for the emission of both charged and neutral particles when muons are captured by aluminium. To investigate this a joint COMET-Mu2E dedicated experiment, ALCAP, is taking place at PSI and an example of preliminary results for neutral particles emitted is shown in Figure 12. The indication so far is that current estimates of backgrounds from this source have been made conservatively.

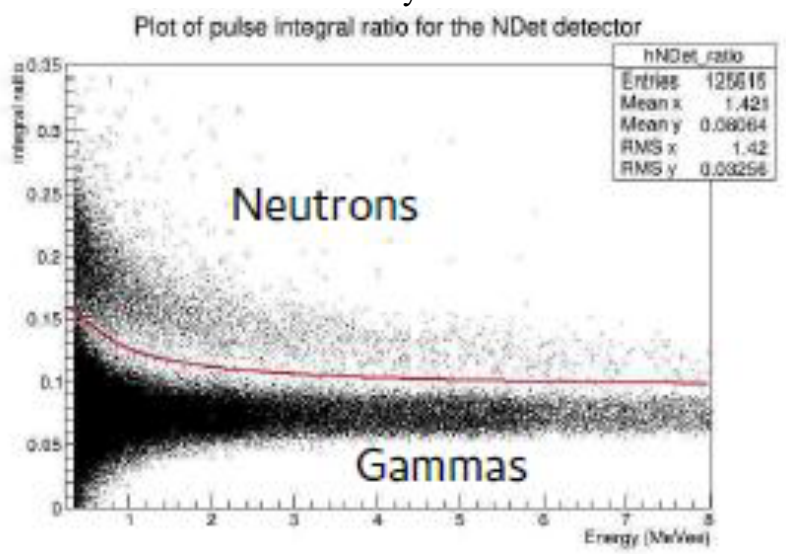

Figure 12. Preliminary results from the ALCAP experiment for neutral particle resulting from muon capture on aluminium

\subsection{Summary of expected backgrounds}

Current estimates of the expected background rates are summarised in Table 1.

Table 1. Summary of estimated background rates

\begin{tabular}{|c|l|}
\hline Beam-related prompt backgrounds & \\
\hline Radiative pion capture & 0.05 \\
\hline Beam electrons & $<0.15$ \\
\hline Muon decay in flight & $<0.0002$ \\
\hline Pion decay in flight & $<0.0001$ \\
\hline Beam-related delayed backgrounds & \\
\hline Neutron induced & 0.021 \\
\hline Delayed pion radiative capture & 0.002 \\
\hline Anti-proton induced & 0.007 \\
\hline Intrinsic physics backgrounds & \\
\hline Muon decay in orbit & 0.15 \\
\hline Radiative muon capture & $<0.001$ \\
\hline$\square^{-}$Capture with neutral emission & $<0.001$ \\
\hline$\square$ Capture with charged emission & $<0.001$ \\
\hline Cosmic ray backgrounds & \\
\hline Cosmic ray muons & 0.002 \\
\hline Electrons from cosmic muons & 0.002 \\
\hline Total & 0.34 \\
\hline
\end{tabular}

\section{Overall Performance}

The expected running time with the $56 \mathrm{~kW}$ beam and predicted sensitivity are given in Table 2
Table 2. Summary of running time and expected sensitivity

\begin{tabular}{|l|c|}
\hline Beam Power at $8 \mathrm{GeV}$ & $56 \mathrm{~kW}$ \\
\hline Running time & $3 \times 10^{7} \mathrm{sec}$ \\
\hline No. of protons & $8.5 \times 10^{20}$ \\
\hline No. of muon stops & $2 \times 10^{18}$ \\
\hline Est. No. of Background & 0.3 \\
\hline Single Event Sensitivity & $2.6 \times 10^{-17}$ \\
\hline Expected limit $(90 \% \mathrm{CL})$ & $6.0 \times 10^{-17}$ \\
\hline
\end{tabular}

\section{Phased Approach}

In an attempt to obtain an earlier result and also to examine in detail the assumptions made on beam quality and backgrounds COMET will run in two phases. The full experiment described above is now termed Phase-II and Phase-I will be a simplified version. The differences are shown in Figure 13.

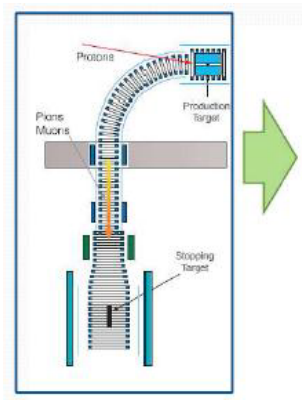

COMET Phase-I

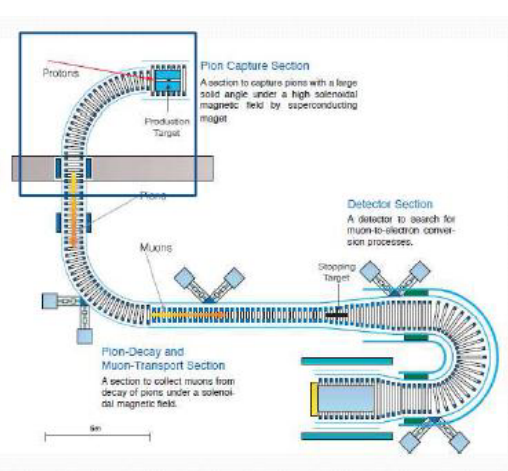

COMET Phase-II
Figure 13. COMET Phase-I and COMET Phase-II

For Phase-I the proton beam power will only be 3.2 $\mathrm{kW}$ and the aim will be to make an initial measurement of $\mu$ to e conversion with a single event sensitivity of $3.10^{-15}$, two order of magnitude better than current limits but two orders of magnitude less than Phase-II. In Phase-I there is no longer a curved solenoid after the muon stopping target and so the electron detection and measurement can no longer be accomplished with an on-axis detector such as the StrECal. Hence for Phase-I the electrons will be detected by a cylindrical drift chamber, CYDET, in a $1 \mathrm{~T}$ solenoid surrounding the stopping target. The StrECal will be used in Phase-I in special runs in order to monitor the beam and backgrounds in preparation for Phase-II.

\subsection{The CYDET}

The detector for Phase-I will be a cylindrical drift chamber in a $1 \mathrm{~T}$ solenoidal field. It is an all stereo chamber with 18 sense wire layers with stereo angles between 64 and $75 \mathrm{mrad}$ giving a longitudinal resolution around $3 \mathrm{~mm}$. The gas will be helium based to minimise multiple scattering although the final mixture awaits prototyping tests. Inside the inner wall of the chamber hodoscopes will provide the trigger for the experiment and $\mathrm{T}_{\text {zero }}$ for the drift measurement. $\mathrm{A}$ 
diagram of this detector for Phase-I is shown in Figure 14.

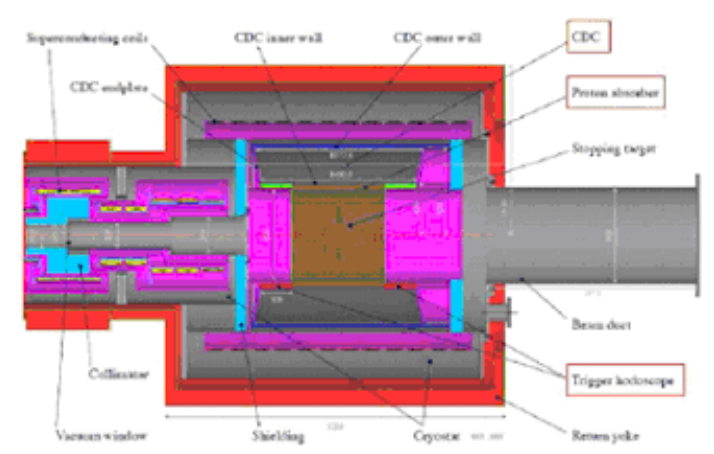

Figure 14. The cylindrical drift chamber and trigger hodoscopes for COMET Phase-I

The CYDET is currently under construction and stringing is in the final stages as can be seen from the photographs in Figure 15.
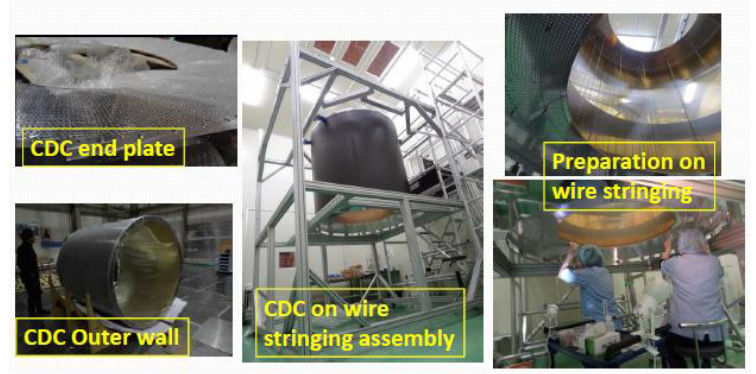

Figure 15. Construction of the CYDET detector

For Phase-I a prototype StrECal is under construction and will be used to monitor the beam and the backgrounds in preparation for Phase-II.

The predicted performance for Phase-I is given in Table 3
Table 3 Predicted running time and performance of COMET Phase-I

\begin{tabular}{|l|c|}
\hline Beam Power at $8 \mathrm{GeV}$ & $3.2 \mathrm{~kW}$ \\
\hline Running time & $9.5 \times 10^{6} \mathrm{sec}$ \\
\hline No. of protons & $2.3 \times 10^{19}$ \\
\hline No. of muon stops & $1.2 \times 10^{16}$ \\
\hline Est. No. of Background & 0.02 \\
\hline Single Event Sensitivity & $3.1 \times 10^{-15}$ \\
\hline Expected limit $(90 \% \mathrm{CL})$ & $7.0 \times 10^{-15}$ \\
\hline
\end{tabular}

\section{Summary}

Muon to electron conversion provides an excellent chance to observe physics beyond the standard model. The COMET experiment is designed to investigate this with the goal of a four order of magnitude improvement on the present limit. The experiment will adopt a phased approach with a first phase to characterise the beams and backgrounds and also make an initial measurement at the $10^{-15}$ level.

The beamline and detectors are currently under construction at J-PARC and the collaborating institutes. Data taking for Phase-I will take place in 2018 with Phase-II to follow two to three years later.

\section{References}

1. A de Gouvea and P Vogel, arXiv:1303.4097 [hep-ph] (2013) 